\title{
Worsening Functional Status in Nephrogeriatrics Needs to Be Accounted for When Clinically Assessing CKD Advancement in Addition to GFR; Supporting Evidence Based on the Practical Application of Theoretical Modelling.
}

Magdalena Wisniewska ( $\sim$ mw3116astra@wp.pl)

Wojskowy Instytut Medyczny

Stanislaw Niemczyk

Wojskowy Instytut Medyczny

Research Article

Keywords: chronic kidney disease, nephrogeriatrics, Tinetti test, Barthel test, IADL test, renal replacement therapy

Posted Date: September 13th, 2021

DOI: https://doi.org/10.21203/rs.3.rs-799342/v1

License: (c) (i) This work is licensed under a Creative Commons Attribution 4.0 International License.

Read Full License

Version of Record: A version of this preprint was published at BMC Geriatrics on July 15 th, 2022. See the published version at https://doi.org/10.1186/s12877-022-03202-4. 


\section{Abstract}

Incidence rates of chronic kidney disease (CKD) are found to increase with age as well as in those elderly patients undergoing renal replacement therapy. Using glomerular filtration rate as a sole predictor of CKD progression in the elderly is highly biased, unless other changes to patient health are accounted for.

Aim: To determine the interrelated dependencies between chronic kidney disease with the functional status of patients aged over 65 years and to elucidate differences in functional status between CKD patients and controls.

Methods: Patient subjects were qualified according to their assessed outcomes from the study protocol, which were achieved by: geriatric interview, assessing functional status by the IADL, Barthel and Tinetti tests together with assessing kidney function by performing laboratory tests of glomerular filtration rate, creatinine and urea. Subjects were divided into two groups: method 1 - according to GFR and method 2 according to GFR and functional test results. The data were statistically analysed by structural equation modelling and k-means.

Results: Positive relationships were found between the CKD stage and comorbidity $(\beta=0.551, p<0.0005)$, along with the number of medications taken and age (respectively $\beta=0.194, p=0.007$ and $\beta=0.301, p$ $<0.0005)$. A highly negative relationship was observed between the CKD stage and the Tinetti test results $(\beta=-0.716, p<0.0005)$, whilst more moderate ones were found with the IADL and Barthel scores (respectively $\beta=-0.49, p<0.0005$ and $\beta=-0.402, p<0.0005$ ). The patient groups demonstrated differences in health status when selected by method- 2 for: age, comorbidity, number of medications taken, fitness test outcomes (Tinetti, Barthel and IADL tests at $p<0.005)$. Those groups divided according to GFR, however only showed differences in age, comorbidity and the number of medication taken ( $p$ $<0.005)$.

Conclusions: The functional status worsens in geriatric patients suffering from CKD. It may thus be important to also account for disruptions to functional status when assessing CKD advancement in the elderly in addition to the GFR. The biggest problems for the over 80 s suffering from CKD are gait and balance disorders, leading to a high risk of falls. Another common problem is polypharmacy, found in both the geriatric population and particularly in those suffering from CKD.

\section{Introduction}

\section{Study context and background}

Diagnosing CKD and monitoring the progression of CKD in the elderly are not only achieved by measuring changes in the levels of biological markers that reflect kidney function (i.e. creatinine, urea and GFR), but also by assessing changes in functional, emotional, cognitive and nutritional status. 
Making such diagnoses requires having the right knowledge and skills to use appropriate scales and tests for performing a comprehensive geriatric assessment. Incidence rates of CKD increases with age and thus becomes a social problem in the elderly. Almost $50 \%$ of people aged over 70 years are diagnosed with CKD grade 3-5 (1).

\section{Study aims}

To determine: 1), the mutually interrelated dependencies between CKD with the functional status of patients aged over 65 years by using the IADL, Barthel, Tinetti tests and 2), to elucidate differences in functional status between CKD patients and controls.

\section{A summary of relevant studies to date}

Patients suffering from advanced CKD will be also frequently suffering from many other diseases, particularly diabetes, cardiovascular disease and vascular diseases of the central nervous system. A high morbidity rate is associated with a high mortality rate in such patients (2). Comorbidity is mentioned by the Bansal risk prediction model, as presented in the ERBP report, where it is one of the predictors determining the probability of death in CKD patients at stages 3-5 who are not undergoing any dialysis therapy (1), (3).

Other studies have also shown that morbidity is associated with polypharmacy and mortality in the elderly (4). Polypharmacy is taken to mean any patient taking more than five drugs, including prescription drugs, as well as the so-called dietary supplements (5). Taking many drugs increases the risk of harmful interactions, including those directed against the kidneys which could thereby lead to acute kidney injury or an exacerbation of CKD.

Physical fitness (functional) of elderly patients is the ability to perform daily life activities, whether independently, with the aid auxiliary devices or from people such as eating and preparing meals, maintaining personal hygiene and that of their surroundings as well as moving around, the ability to take medications independently, using the telephone and exercising personal control over finances. Progressive aging however leads to a gradual loss of both fitness and being able to independently execute daily activities. A comparative study has shown that the ability of being able to carry out complex everyday activities (Instrumental Activity of Daily Living; IADL) had decreased to $9.9 \%$ in 90 -year-olds compared to rates of $79.4 \%$ in those aged $65-69$ years (6). Moreover, assistance is needed by $1: 10$ persons aged above 80 years in performing basic self-activities, such as personal hygiene and walking or eating ( $A D L)$; indeed, more often than not by women than men (7). This however relates to the general population of the elderly without CKD, but there are haven't been any studies in this area on people with renal insufficiency. Mobility problems (e.g. gait and balance) contribute to falls, which affects $30-50 \%$ of the elderly living outside of care institutions, with half having falling-accidents more than once yearly (8). The 2016 ERBP report states that CKD is associated with patients' state of physical fitness and frailty syndrome, and therefore with the level of exercises performed and mobility (1). 


\section{Reasons why the study is important.}

The presented study emphasises the role of undertaking a comprehensive geriatric assessment, particularly to detect changes in the patient's functional status when assessing CKD advancement. It also suggests a reversal from the current trend of relying only on laboratory parameters in such an assessment. This research is also highly relevant and needed because there are as yet still no guidelines for medical practitioners in geriatric nephrology on which is the most appropriate tool for assessing functional status in the elderly suffering from CKD.

\section{Methods}

\section{Study protocol}

The study subjects were assessed according to the developed protocol, which included the following procedures:

1. Geriatric interview including, inter alia, age, comorbidity (number of diseases), the recorded history of medications taken.

2. Geriatric physical examination, including:

Assessing the patient's functional status using the Lawton IADL test , the Barthel test and the Tinetti test to assess the risk of falls.

3. Additional blood tests for creatinine, urea and GFR assessment (short version MDRD).

The scoring scales assessing functional status by the IADL, Barthel, Tinetti methods can be found in the footnotes at the end of this paper.

\section{Characteristics of the study subjects}

The study group were patients aged over 65 years of age, hospitalised at the Nephrology Clinic of the Military Institute of Medicine and those patients under long-term care at the Dialysis Center, also from the Military Institute of Medicine.

The participants were divided into groups by using two methods: 1) according to GFR division and, 2) according to division by GFR and functional test results.

The following groups were obtained when the GFR criterion was used:

- Group-1, the haemodialysis, with CKD stage V (GFR of $\left.<15 \mathrm{ml} / \mathrm{min} 1.73 \mathrm{~m}^{2}\right) ; \mathrm{n}=28$.

- Group-2, with CKD stage III-IV (GFR of $\left.15-60 \mathrm{ml} / \mathrm{min} / 1.73 \mathrm{~m}^{2}\right) ; \mathrm{n}=62$.

- Group-3, controls, subjects without CKD, $\mathrm{n}=23$, (patients with a $\quad$ GFR of $>60 \mathrm{ml} / \mathrm{min}$ were qualified for this study, without any past history of CKD nor with any additional tests suggesting 
a past CKD diagnosis).

The following groups were obtained when the GFR criterion was used together with functional test outcomes:

- Group i.1 with CKD stage IV-V, $n=28$, who were on haemodialysis together with $n=16$ subjects with CKD stage IV that had similar functional test results; giving a total of $n=44$.

- Group i.2 with CKD stage II-III and similar functional test results; $\mathrm{n}=34$.

- Group i.3, the controls ( $n=23$ CKD-absent and $n=12$ subjects with a $\quad$ GFR of $50-59 \mathrm{ml} / \mathrm{min}$ that had similar functional test outcomes to those with CKD absent); giving a total of $n=35$.

The group breakdowns distinguished 3 clusters of patients, corresponding to the 3 patient groups so formed by the primary division according to the GFR described earlier: the CKD group, the hemodialysis group and the control group. Similar results were observed for each group in terms of both renal function markers and the geriatric evaluation tests.

\section{Statistics methods}

A hypothetical model was created for this study, which was based on the literature, that presents the interrelationship between CKD (latent variable) and functional status (endogenous variables) of patients with their age, number of medications taken and comorbidity (exogenous variables). This structural model is shown in Figure 1.

Structural equations were used to verify the model and how well the obtained data fitted in using the ADF (Asymptotically Distribution Free estimator) (9). Using parametric statistics were precluded because the data were not normally distributed and therefore cluster analysis ( $k$-means method) was used enabling similar clusters to be distinguished according to the intensity of the variables (10). The selected groups were compared with each other using ANOVA (11).

\section{Results}

\section{Theoretical model}

Positive relationships were found between the CKD stage and comorbidity $(\beta=0.551, p<0.0005)$, the number of medications taken $(\beta=0.194, p=0.007)$ and age $(\beta=0.301, p<0.0005)$. Whereas a highly negative relationship was observed between the CKD stage and outcome from the Tinetti test $(\beta=-0.716$, $p<0.0005$ ), whilst the IADL and Barthel scores showed more moderate levels at respectively $\beta=-0.49, p$ $<0.0005$ and $\beta=-0.402, p<0.0005$.

\section{Group results}

\section{Group outcomes divided according to GFR and other variables}


Three similar clusters of test subjects were distinguished according to the intensity of variables as described in the theoretical model. The first group consisted of 44 subjects who had the highest concentrations of creatinine (normalised mean value) and urea but the lowest GFR i.e. Those with advanced CKD stage IV-V. This group had the lowest mean age among all the groups distinguished but the highest comorbidity and they took the most drugs. Outcomes from the Tinetti test were average, whilst they were high for everyday activities (according to the IADL and Barthel tests) (Figure 2, Table 1).

Table 1. Cluster group arithmetic mean values according to GFR and function tests

\begin{tabular}{llll} 
& HD & CKD & Control \\
\hline Creatinine & 4.98 & 2.13 & 1.07 \\
\hline Urea & 129.45 & 92.111 & 43.49 \\
\hline GFR & 13.43 & 34.39 & 69.77 \\
\hline Age & 72.43 & 84.64 & 76.8 \\
\hline Comorbidities & 5.22 & 4.55 & 2.8 \\
\hline Medication & 10.15 & 7.02 & 5.42 \\
\hline Tinetti & 22.65 & 17.38 & 26.6 \\
\hline IADL & 7.54 & 5.5 & 7,6 \\
\hline Barthel & 97.5 & 82.05 & 99.14 \\
\hline Number of cases & 44 & 34 & 35 \\
\hline Percentages (\%) & 38.93 & 30.08 & 30.97
\end{tabular}

A second cluster was classified by algorithms into 34 people with average study levels of creatinine, urea and GFR; this being subjects with stage II-III CKD (Figure 2,Table 1). This group had the highest mean study age, the highest score comorbidity ascores of all groups, medication at average study levels and had the lowest Tinetti test scores as well as on the daily activities tests (IADL, Barthel).

The algorithms classified 35 subjects in the third cluster with the lowest creatinine and urea concentrations but the highest GFR value. These were subjects without CKD (Figure 2, Table 1). They had average ages compared to the other groups, the lowest comorbidity and took the least drugs, achieving the highest outcomes in the Tinetti test as well as the highest scoring of daily activities by the I-ADL and Barthel tests.

These clusters so identified showed statistically significant differences with in all variables (Table 2). Large effects between the clusters were observed for creatinine, urea, GFR, age, comorbidity, medications and the Tinetti, IADL and Barthel test scores. There were significantly differences in all variables between the identified; 
Table 2. ANOVA performed on variables between groups divided according to GFR and function testing.

\begin{tabular}{lllllllll} 
& $\begin{array}{l}\text { Intra-group } \\
\text { variation }\end{array}$ & $\mathrm{f}$ & $\begin{array}{l}\text { Inter-group } \\
\text { variation }\end{array}$ & $\mathrm{df}$ & $\mathrm{F}$ & $\begin{array}{l}\mathrm{p} \text { - } \\
\text { value }\end{array}$ & $\mathrm{\eta}^{2}$ & Int. \\
\hline Creatinine & 329.1 & 2 & 188.6 & 10 & 95.9 & $<0.005$ & 0.635 & Large \\
\hline Urea & 144064.9 & 2 & 122889.8 & 10 & 64.4 & $<0.005$ & 0.539 & Large \\
\hline GFR & 62245.8 & 2 & 23286.0 & 10 & 147.0 & $<0.005$ & 0.727 & Large \\
\hline Age & 2883.9 & 2 & 3752.2 & 10 & 42.2 & $<0.005$ & 0.434 & Large \\
\hline Comorbidities & 118.8 & 2 & 469.7 & 10 & 13.9 & $<0.005$ & 0.201 & Large \\
\hline Medication & 461.6 & 2 & 909.4 & 10 & 27.9 & $<0.005$ & 0.336 & Large \\
\hline Tinetti & 1475.0 & 2 & 3252.3 & 10 & 24.9 & $<0.005$ & 0.312 & Large \\
\hline IADL & 101.9 & 2 & 241.8 & 10 & 23.1 & $<0.005$ & 0.296 & Large \\
\hline Barthel & 6267.0 & 2 & 33505.2 & 10 & 10.2 & $<0.005$ & 0.157 & Large
\end{tabular}

Description of symbols used in Tables 2 \& 4 (and in text): $\mathrm{df}=$ degrees of freedom (either between or within groups), $\mathrm{F}=$ variance ratio, $\eta^{2}=$ a measure of the proportion of total variance (related to the effect size index), Int = the magnitude of the effect size index, ie.; 0.01-0.06 (Small), 0.06-0.14 (Medium) and $>0.14$ (Large), (29).

\section{Group outcomes divided according to GFR}

A cluster analysis was also performed on the variables described in the theoretical model by using the kmeans method where a modelled grouping of variables was introduced for those undergoing dialysis, with and without renal failure, in such a way that only subjects belonging to groups selected on the basis of eGFR values were classified to the clusters.

This aimed to determine whether there are significant differences between these groups and what profile is characteristic for each of these groups, in terms of the variables described in the theoretical model. The solution to the cluster analysis is presented in Figure 3.

People belonging to the group with renal insufficiency ( $n=62$, Table 3 ) have average results among all groups in terms of creatinine, urea and GFR, they are characterized by the highest mean age, lower than the group of patients on hemodialysis ( $n=28$, Table 3 ) comorbidity and the number of drugs taken. The remaining differences between the groups were statistically insignificant (Table 4).

Control subjects, without CKD ( $n=23$, Table 3$)$, had the lowest creatinine and urea, whilst having the highest GFR. They were of average study age, had the lowest comorbidity index and took the fewest drugs. All other differences found between the groups were statistically insignificant (Table 4).

Table 3. Cluster means in groups according to GFR 


\begin{tabular}{llll} 
& HD & CKD & Control \\
\hline Creatinine & 5.87 & 2.31 & 0.92 \\
\hline Urea & 130.35 & 93.98 & 37.96 \\
\hline GFR & 10.35 & 32.62 & 82.17 \\
\hline Age & 71.71 & 80.40 & 76.52 \\
\hline Comorbidities & 5.28 & 4.45 & 2.56 \\
\hline Medication & 10.78 & 7.19 & 5.56 \\
\hline Tinetti & 21.89 & 21.66 & 24.47 \\
\hline IADL & 7.25 & 6.66 & 7.34 \\
\hline Barthel & 96.78 & 91.53 & 94.13 \\
\hline Number of cases & 28 & 62 & 23 \\
\hline Percentages (\%) & 24.77 & 54.86 & 20.35
\end{tabular}

The differences between groups were large for creatinine, urea, GFR, age, comorbidity, and medications, but small for Tinetti test outcomes and those measuring daily activities (ie. I-ADL and Barthel tests) (Table 4).

Table 4. ANOVA performed on variables between groups divided according to GFR.

\begin{tabular}{lllllllll} 
& $\begin{array}{l}\text { Inter-group } \\
\text { variation }\end{array}$ & $\mathrm{df}$ & $\begin{array}{l}\text { Intra-group } \\
\text { variation }\end{array}$ & $\begin{array}{l}\mathrm{D} \\
\mathrm{f}\end{array}$ & $\mathrm{F}$ & $\begin{array}{l}\mathrm{p}- \\
\text { value }\end{array}$ & $\eta^{2}$ & Int. \\
\hline Creatinine & 358.8 & 2 & 158.9 & 110 & 124.19 & $<0.005$ & 0.693 & Large \\
\hline Urea & 108575.5 & 2 & 158379.2 & 110 & 37.70 & $<0.005$ & 0.407 & Large \\
\hline GFR & 67994.3 & 2 & 17537.5 & 110 & 213.23 & $<0.005$ & 0.795 & Large \\
\hline Age & 1481.7 & 2 & 51544 & 110 & 15.81 & $<0.005$ & 0.223 & Large \\
\hline Comorbidities & 97.8 & 2 & 490.7 & 110 & 10.95 & $<0.005$ & 0.166 & Large \\
\hline Medication & 387.0 & 2 & 984.0 & 110 & 21.63 & $<0.005$ & 0.282 & Large \\
\hline Tinetti & 139.1 & 2 & 4588.3 & 110 & 1.66 & 0.193 & 0.029 & Small \\
\hline IADL & 11.3 & 2 & 332.4 & 110 & 1.87 & 0.158 & 0.033 & Small \\
\hline Barthel & 549.4 & 2 & 39222.8 & 110 & 0.77 & 0.465 & 0.014 & Small
\end{tabular}

Description of symbols used in Tables 2 \& 4 (and in text): $\mathrm{df}$ = degrees of freedom (either between or within groups), $\mathrm{F}=$ variance ratio, $\eta^{2}=$ a measure of the proportion of total variance (related to the effect 
size index), Int = the magnitude of the effect size index, ie.; 0.01-0.06 (Small), 0.06-0.14 (Medium) and $>0.14$ (Large), (29).

\section{Discussion}

\section{Relationship of chronic kidney disease with functional status}

The analysis using structural equations demonstrated the different extents by which CKD is associated with all the studied variables in the elderly (i.e. numbers of medications taken, comorbidity, age and physical fitness).

As an independent factor, having CKD worsens the physical condition of elderly patients and results in a two-fold increase in the risk of impaired functional status compared to those elderly people without CKD (12), (13), (14), (15), (16) .

The mechanisms underlying this relationship are complex. Some studies have associated CKD with increased comorbidity, especially with cardiovascular diseases, which in turn reduces physical activity and thereby an impaired functional status (17). Indeed, our study showed that CKD is moderately associated with comorbidity and patient performance in functional tests. Other studies have explained this association by the presence of the so-called fragility syndrome which occurs three times more often in patients with CKD and end-stage renal disease than subjects with normal renal function (13), (18), (19). The fragility phenotype is characterised by a tendency for unintentional weight loss, muscle weakness, subjective exhaustion, slow walking speed and decreased physical activity (20). One of its causes is considered to be high comorbidity. It is also responsible for increasing the body's energy consumption thereby leading calorific deficiency and, consequently to protein-energy malnutrition. Other causes of the fragility syndrome in CKD include anaemia, chronic inflammation, metabolic acidosis, endocrine and calcium-phosphate disorders (21). Remedying such disorders improves the functional status in postkidney transplant patients and is another piece of evidence to show that CKD worsens the functional status of patients (22).

The relationship between CKD and patient age is unclear. A multicentre meta-analysis by S. Hallan (JAMA in 2012), demonstrated that low eGFR and high albuminuria are associated with mortality and ESRD (End-Stage Renal Disease) irrespective of age (26). Nonetheless, our study in fact showed an association of CKD with patient age. Reasons for this disagreement are most likely because mean age in the former study was 49.4 years compared to our study mean of 80.4 years.

The problem of polypragmasy is common among older patients, but there are only a few scientific reports regarding any direct relationship between the number of medications taken with CKD. The presented study however showed a weak relationship between CKD and the number of medications taken, whereas an English study showed that approximately $20 \%$ of patients with CKD take more than 10 different medications daily (27). 
Our study has revealed that the patient groups with renal failure, without renal failure and haemodialysis significantly differed from each other in terms of: age, comorbidity, number of medications taken and fitness test outcomes (Tinetti test, Barthel's scale, IADL scale); $p<0.005$. As expected, the control group outcomes showed the lowest comorbidity, patients taking the least drugs, highest scoring in gait and balance tests (Tinetti test) along with the fitness tests (IADL, Barthel).

Haemodialysis patients are however younger, have the highest comorbidity, take the most medications, and have average study results in for the fitness tests. The average study outcomes in tests of daily activities in this group can be explained by their lower age compared to the ages of the CKD subjects (72.4 years vs 84 years). Nevertheless, the high scores from the IADL test (7.5 pp. / 8 pp.) and Barthel test (97.5 pp. / 100 pp.) did not correlate with the low score in the Tinnetti test (22.6 pp. / 28 pp.). These results suggest there is an additional factor present that increases the risk of falls within this patient group; this might be the large number of drugs taken (average of $10 /$ day) or the high concentrations of creatinine (average $4.98 \mathrm{mg} / \mathrm{dl}$ ) or urea (average $129.45 \mathrm{mg} / \mathrm{dl}$ ).

The CKD group have the highest mean age (84 years), take less drugs than dialysis patients, and have a lower comorbidity compared to dialysis patients. Furthermore, they scored the lowest in the gait and balance test (17.3p. / 28p in the Tinetti test) and also the lowest results in the fitness tests (IADL test, Barthel). Such low scoring in the functional tests are due to the presence of advanced CKD (stage III) and the age of the respondents. Similar observations have been presented by D. Weiner and S. Seliger (28). One can assume that the low scores obtained by patients aged over 80 years in the Tinetti test will lead to more frequent falls in these patients. Indeed, yearly falls are observed in around $30 \%$ of people aged over 75 years without CKD, in about $38 \%$ of people with CKD and about $50 \%$ of patients on haemodialysis (23), (24), (25). Disorders in gait arise from the following in these patients: weakening of muscle strength when suffering from the fragility syndrome, sensation impairment furing diabetic neuropathy, visual disturbances whilst suffering from cataracts, glaucoma, macular degeneration, diabetic and hypertensive retinopathy, disorders of the vestibular system (caused by hyper- and arterial hypotension), water and electrolyte disruption, polypragmasy and cerebral circulatory disorders.

Both CKD patient groups, (i.e. stage III of CKD and the HD - stage IV-V of CKD) gave more inferior test scores for the functional test compared to controls thus providing evidence that CKD significantly worsens the functional status of patients irrespective of age.

\section{Differences in patients' groups health status divided according to GFR}

Our results demonstrate that those subject groups with renal failure, without renal failure and those undergoing haemodialysis significantly differ from each other in terms of: age, comorbidity and number of medications taken; $p<0.005$. Differences in daily activities were found to be insignificant as measured by the Tinetti, IADL and Barthel scales which shows that assessing the severity and progression of CKD should be made not only on the basis of eGFR values, but also by assessing changes in the functional status of patients. 
Finally, the amount of medications taken by the patient groups also needs commenting on. The most drugs were taken by the HD patients $(\sim 10)$, regardless of group breakdown, whilst slightly fewer were taken by CKD patients $(\sim 7)$, and the least by the control group $(\sim 5)$. This means that the problem of polypragmasy concerned all subjects and therefore it is necessary to take remedial measures to reduce this unfavourable phenomenon.

\section{Conclusion}

CKD in the geriatric population worsens their functional status. Disruptions to the functional status of such patients may also play a role when assessing the advancement of CKD in the elderly, in addition to the GFR value. Gait and balance disorders were found to be the biggest problems in the CKD group aged over 80 years resulting in a high risk of falls. Polypharmacy is also another common problem in the elderly, especially in patients with CKD.

\section{Abbreviations}

CKD - Chronic Kidney Disease

GFR - Glomerular Filtration Rate

IADL - Instrumental Acivities of Daily Living

ERBP - European Renal Best Practice

MDRD - Modification of Diet in Renal Disease

\section{Declarations}

\section{Data, materials used}

https://doi.org/10.1111/j.1532-5415.1986.tb05480.x

https://www.leadingagemn.org/assets/docs/Tinetti-Balance-Gait--POMA.pdf

https://doi.org/10.1093/geront/9.3_Part_1.179

https://www.alz.org/careplanning/downloads/lawton-iadl.pdf

http://www.sciepub.com/reference/163871

https://stopstroke.massgeneral.org/pdfs/barthel_reprint.pdf

\section{Availability of data and materials}


The raw data of the current study are not publicly available due to the protection of the participants personal information but are available from the corresponding author on reasonable request.

\section{Ethical approval and consent to participate}

This study was performed in accordance with the Helsinki Declaration. The Bioethics Committee of the Military Medical Institute in Warsaw approved the study (Resolution No. 69 / WIM / 2015). All participants obtained written informed consent prior to taking part in the interview.

\section{Consent for publication}

All authors have read and approved the final draft of this manuscript and agree to its submission.

\section{Competing interests}

The authors declare none.

\section{Funding}

The project was financed by the Military Medical Institute in Warsaw as part of its own statutory operational remit (Project No. 439, Decision No. 33 / W / 2016). The funding body did not have any involvement in the study design, methods, data collection or analysis, nor did it have any involvement in preparing the manuscript.

\section{Authors' contribution}

The main contractor of the research project and the author of the article; MW; the originator of the research project, substantive supervision over the project and article, SN. All authors have read and approved the final manuscript.

\section{Acknowledgments}

Not applicable

\section{Author details:}

${ }^{1}$ Department of Internal Diseases, Nephrology and Dialysis Therapy, Military Institute of Medicine, 04-141 Warsaw, Szaserow 128 Street, Poland

\section{References}

1. ERBP: Clinical Practice Guideline on management of older patients with chronic kidney disease stage $3 \mathrm{~b}$ or higher (eGFR<45ml/min). Nephrol Dial Transplant. 2016;31 Suppl 2:1-66.

2. Kyeong MK, Hyung JO, Dong-Ryeol R. Impact of chronic kidney disease on mortality: A nationwide cohort study. Kidney Res Clin Pract. 2019;38(3):382-90. 
3. Bansal N, Katz R, De Boer IH, Peralta CA, Fried LF, Siscovick DS, Ri in DE H, C, Cummings SR, Harris TB, Kritchevsky SB, Sarnak MJ, Shlipak MG IJ. Development and validation of a model to predict 5year risk of death without ESRD among older adults with CKD. Clin J Am Soc Nephrol. 2015;10:36371.

4. Hajjar R, Cafiero AC, Hanlon JT. Polypharmacy in elderly patients. Am J Geriatr Pharmacother. 2007;5(4):345-51.

5. Byliniak M, Tuszyński K, Czech M, Kardas P. NFZ o zdrowiu. Polipragmazja. 2020.

6. Andersen-Ranberg K. Trends Over Time in Cognitive Function and Activities of Daily Living Among Danish Centenarians. Annu Rev Gerontol Geriatr. 2013;33(1):333-59.

7. Liu, C., Leung, D., Chi I. Social functioning, polypharmacy and depression in older Chinese primary care patients. Aging Ment Heal. 2011;15(6):732-41.

8. Wojszel ZB. Geriatryczne zespoły niesprawności i usługi opiekuńcze w późnej starości. Trans Humana; 2009.

9. Szymańska A. Założenia formalne modeli weryfikowanych za pomocą układów równań strukturalanych. Stud Psychol (Bratisl). 2016;16(2):93-116.

10. Nisbet R, Elder J, Miner G. Handbook of statistical analysis and data mining applications. Elsevier; 2009.

11. Tabachnick B, Fiddell L. Experimental designs using ANOVA. Northridge: California State University; 2020.

12. Roderick PJ, Atkins RJ, Smeeth L et al. Detecting chronic kidney disease in older people; what are the implications? Age Ageing. 2008;37(2):179-86.

13. Shlipak MG, Stehman-Breen C, Fried LF, Song X, Siscovick D, Fried LP, et al. The Presence of Frailty in Elderly Persons with Chronic Renal Insufficiency. Am J Kidney Dis. 2004;43(5):861-7.

14. Bowling CB, Sawyer P, Campbell R., Ahmed A, Allman RM. Impact of Chronic Kidney Disease on Activities of Daily Living in Community-Dwelling Older Adults. Journals Gerontol. 2011;66A(6):68994.

15. Smyth A, Glynn LG, Murphy AW, Mulqueen J, Canavan M, Reddan DL, et al. Mild Chronic Kidney Disease and Functional Impairment in Community-Dwelling Older Adults. Age Ageing. 2013;42(4):488-94.

16. Walker SR, Gill K, McDonald K, Komenda P, Rigatto C. Association of frailty and physical function in patients with non-dialysis CKD: a systematic review. BMC Nephrol. 2013;14(228).

17. Anand S, Johansen KL, Tamura MK. Aging and Chronic Kidney Disease: The Impact on Physical Function and Cognition. Journals Gerontol. 2014;69A(3):315-22.

18. Roshanravan B, Khatri M, Robinson-Cohen C, Levin G, K.V. P., De Boer, I.H. et al. A prospective study of frailty in nephrology-referred patients with CKD. Am J Kidney Dis. 2012;60:912-21.

19. Johansen KL, Chertow GM, Jin C, Kutner NG. Significance of frailty among dialysis patients. J Am Soc Nephrol. 2007;18(2960-2967). 
20. Fried LP, Tangen CM, Walston J, Newman AB, Hirsch C, Gottdiener $\mathrm{J}$ et al. Frailty in older adults: evidence for a phenotype. J Gerontol. 2001;56:146-56.

21. Lam M, Jassal SV. The Concept of Frailty in Geriatric Chronic Kidney Disease (CKD) Patients. Blood Purif. 2015;39:50-4.

22. De Mendonca AEO, De Vasconcelos Torres G, De Góes Salvetti M, Alchieri JC, Costa IKF. Changes in Quality of Life after kidney transplantation and related factors. Acta Paul Enfermagem- line. 2014;27(3).

23. Polinder-Bos HA, Emmelot-Vonk MH, Gansevoort RT, Diepenbroek A, Gaillard C. High Fall Incidence and Fracture Rate in Elderly Dialysis Patients. Neth J Med. 2014;72(10):509-15.

24. De Carvalho TC, Dini AP. Risk of falls in people with chronic kidney disease and related factors. Rev Latino-Americana Enfermagem- line. 2020;

25. Morley JE. Chapter 33. Falls in Elderly Patients With Kidney Disease. In: Nephrology TAS of, editor. Geriatric Nephrology Curriculum. 2009. p1-5.

26. Hallan S, Matsushita K and for the CPC. Age and the Association of Kidney Measures with Mortality and End-Stage Renal Disease. JAMA. 2012;308(22):2349-60.

27. Parker K, M. N, Jayanti A et al. Medication burden in CKD-5D: impact of dialysis modality and setting. Clin Kidney J. 2014;7:557-561.

28. Weiner DE, Seliger SL. Cognitive and Physical Function in Chronic Kidney Disease. Curr Opin Nephrol Hypertens. 2014;23(3):291-7.

29. Cohen J. The analysis of variance and covariance. In: Statistical power analysis for the behavioral sciences. Second. New York: Lawrence Erlbaum Associates; 1988. p. 285-7.

\section{Figures}




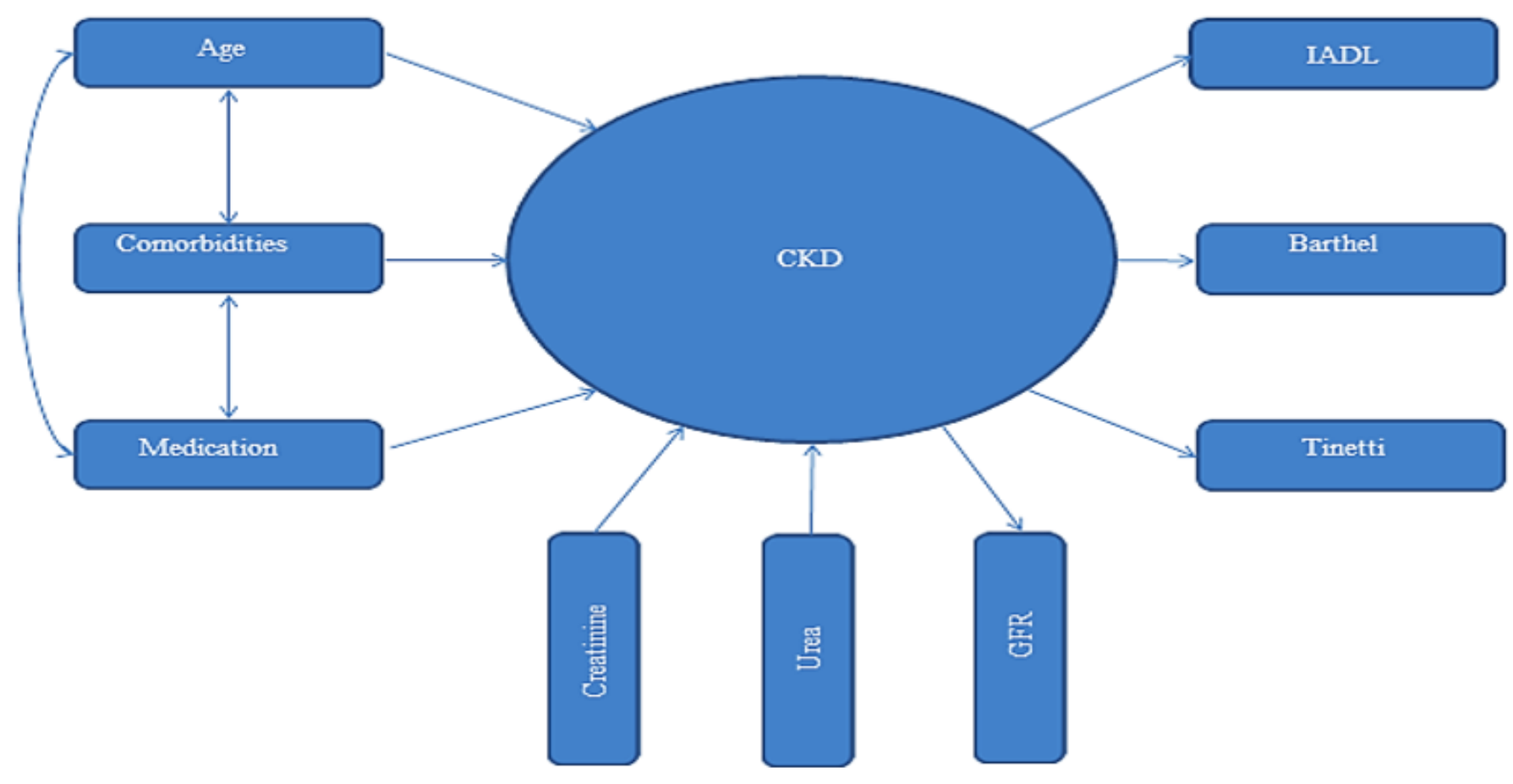

Figure 1

The theoretical model in the author's own elaboration describing the interrelationships between CKD and the patient's age, comorbidity, the number of medications taken and the functional status measured using the IADL, Tinetti, and Barthel tests. 


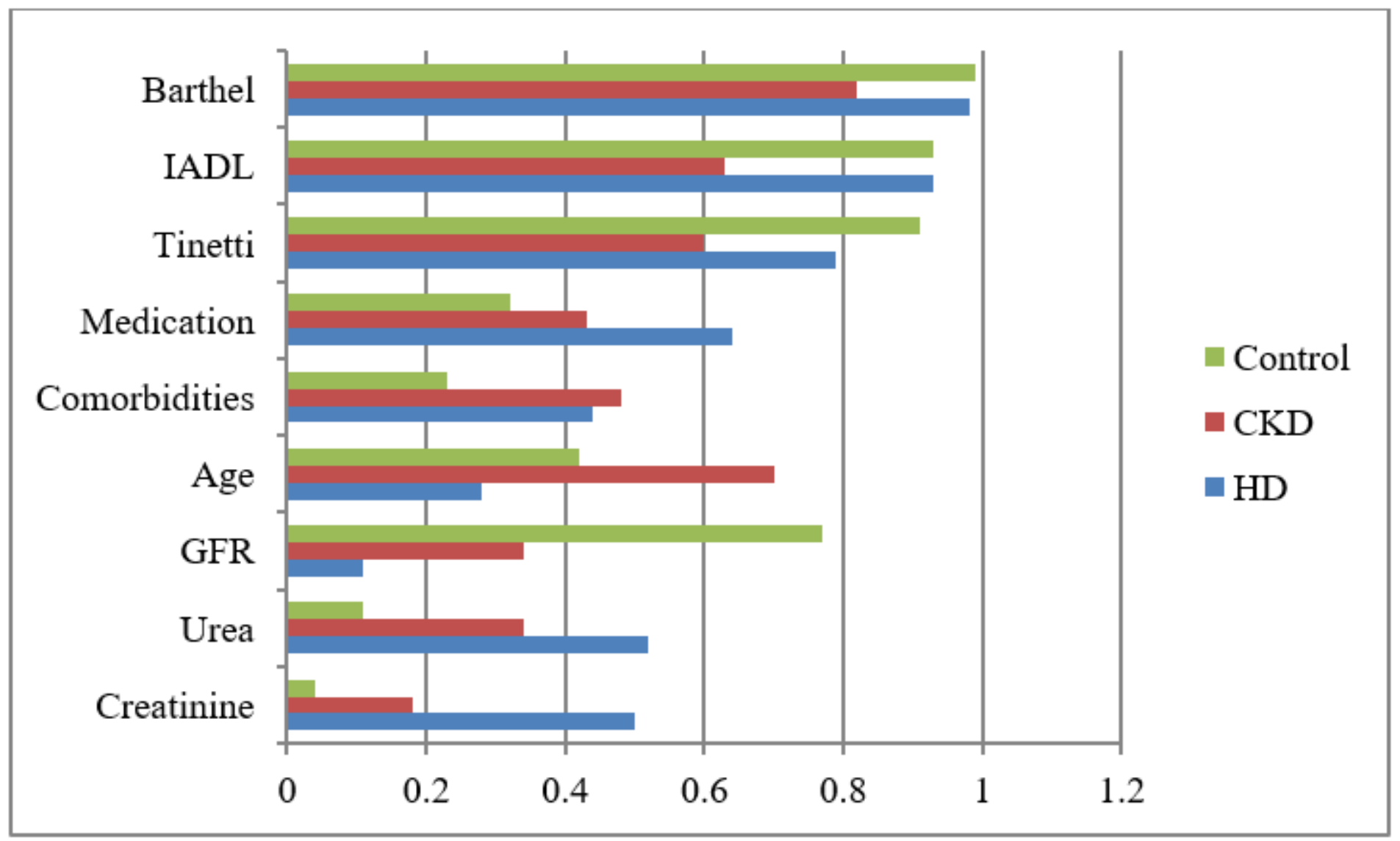

Figure 2

Normalised variable means according to GFR and function tests. Legend: HD group (i.1 group), CKD group (i.2 group), Control group (i.3 group) Y-axis: Change to decimal point notation, ie. 1.2, 1.0, 0.8, 0.6, 0.4, 0.2 \& 0.0 X-axis: Creatinine, Urea, GFR, Age, Comorbidities, Medication, Tinetti, IADL, Barthel 


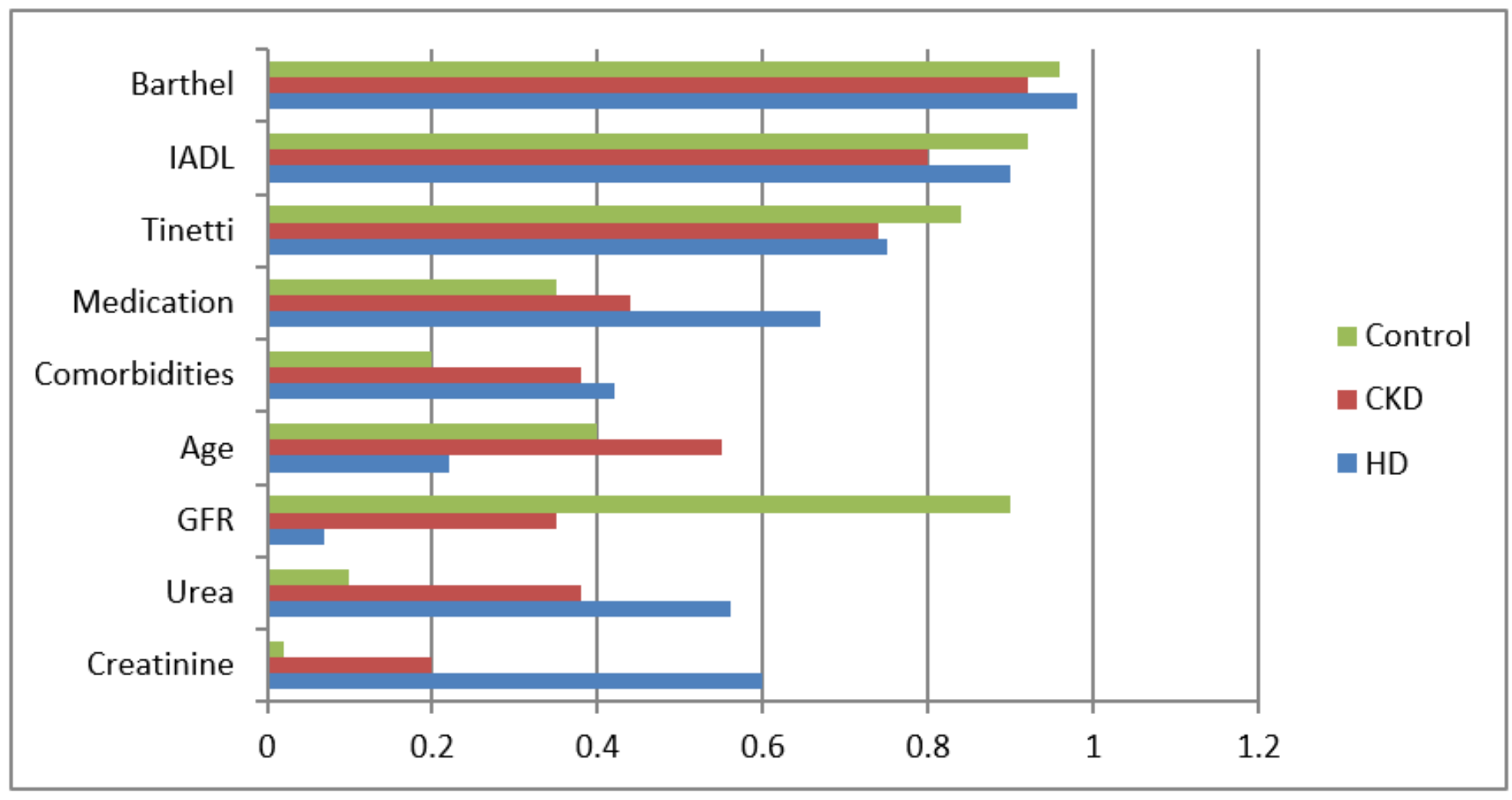

\section{Figure 3}

Grouped clusters developed by the k-means method according to GFR. Legend: HD (group 1), CKD (group 2), Control (group 3) Y-axis: Change to decimal point notation, ie. 1.2, 1.0, 0.8, 0.6, 0.4, 0.2 \& 0.0 X-axis: Creatinine, Urea, GFR, Age, Comorbidities, Medication, Tinetti, IADL, Barthel 